\section{DNA and aging}

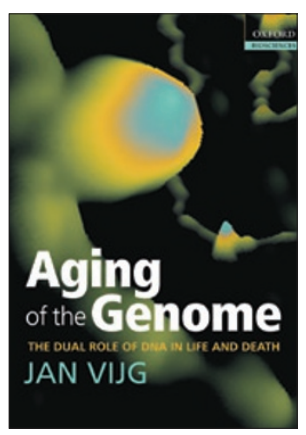

\section{Aging of the Genome: The Dual Role of DNA in Life and Death}

By Jan Vijg

Oxford University Press, 2007

372 pp., paperback, $\$ 65.00$

ISBN 978-0-19-856923-7

\section{Reviewed by Vilhelm A Bohr \& Christina T Bohr}

One of the major theories of aging, proposed by Harman in 1956, suggests that it is caused by the accumulation of oxidative damage affecting macromolecules, DNA, RNA and proteins, and possibly cellular organelles and membranes. Although there are many other theories of aging, this has been one of the most persistent. There has been much discussion of the theory over the years, and it has stimulated extensive experimental work. Although damage to all macromolecules can be deleterious and significant, it has often been speculated that DNA damage is of particular importance, as this is the master molecule, whereas the other macromolecules are more readily renewable. Deficient RNA and protein can be replaced, but damage to DNA can cause problems with replication, transcription and signaling, and changes in these events are characteristics of the aging process.

In his book, Jan Vijg gives a very comprehensive and well-composed view of how the changes in DNA can be a causative factor in aging, but still with a view to various other theories of aging. There is an interesting and illuminating description of the history and developments of modern gerontology and of the way these advances have gone hand in hand with the great advances in understanding the genome. Vijg gives an excellent, easily readable and understandable discussion of DNA damage and the maintenance process of DNA repair. He then describes advances in understanding genetic disorders associated with accelerated aging and the ways that mouse models deficient in these disease genes have provided additional insight because of the resulting changes in phenotype. Vijg's own work has been in the area of understanding mutations, and the insight he provides in this area is especially enlightening. In particular, he has developed a mouse model with a marker gene inserted that can be used to detect mutations in various organs. This has been very useful for determining relative mutation frequencies in tissues and how they change with aging and in conjunction with deficiencies in DNA repair.

There are two separate genomes in human cells: the nuclear and the mitochondrial. The importance of the mitochondrial genome is often overlooked. Vijg describes this genome and discusses the significance of DNA damage to mitochondria, which may be an important signaling factor in the aging process. A leading theory of aging, which he mentions but does not extensively discuss, is that DNA damage accumulates in the mitochondria with aging as a result of defective DNA repair mechanisms, and the mitochondria then deteriorate and cause general decline.

The telomeres, or ends of the chromosomes, are a very important part of the genome and are essential for genome stability. These ends shorten with age, a process that may represent an important clocklike mechanism for age-associated phenomena and disease. Although this topic is discussed in the book, it could have had a more prominent position given how central it is to the understanding of aging and given the many recent advances in characterizing the proteins involved in telomere maintenance and their mechanisms of action.

The concepts presented are written in a very accessible fashion, and many experiments are described to explain the basis of our current understanding. The explanations are detailed and employ a higher level of scientific terminology than is used to explain each preceding main concept. Some readers may therefore not be able to follow the experimental procedures unless they have firm grounding in the research involved. Fortunately, Vijg includes supplements to the text to aid the reader. After many of the sections, he concludes with a brief, simple summary to emphasize the main concepts discussed. He also provides useful illustrations throughout, some representing figures from published work and others emphasizing important points with nice sketches. Particularly in the area of DNA damage, repair and mutations, there are some quite informative illustrations. The glossary is a bit short, but the list of references is extensive and sufficient.

In sum, this book should appeal to a readership that includes scientists in related areas and also university-level students with a scientific major, especially those considering a career in the biological sciences. The book represents a substantial effort and documents the broad and deep insight that Vijg has into the field of aging. It should greatly stimulate interest and the entry of promising new scientists into the field and will help heighten the profile and importance of aging research in general. 